\title{
A MODIFIED TWO-SCALE MICROWAVE SCATTERING MODEL FOR A GAUSSIAN- DISTRIBUTED CONDUCTING ROUGH SURFACE
}

\author{
F. Yu ${ }^{1}$, H. Wang ${ }^{1}$, Z. Y. Chen ${ }^{2}$ \\ ${ }^{1}$ Chinese Academy of Surving and Mapping, 100830, NO. 28 Lianhuachixi Road, Beijing China- (yufan021@126.com, \\ Wanghao@casm.ac.cn) \\ 2 Chaoyang Campus of Tsinghua University Affiliated High School, 100027, NO.21 Xinyuan Street, Beijing, china- \\ chenzhangyue@126.com
}

Commission WG III/2

KEY WORDS: Two-scale Model; Rough Surface; Backscattering Model; Wavelet Packet Transform; AIEM

\begin{abstract}
:
A modified two-scale microwave scattering model (MTSM) was presented to describe the scattering coefficient of natural rough surface in this paper. In the model, the surface roughness was assumed to be Gaussian so that the surface height $z(x, y)$ can be split into large-scale and small-scale components relative to the electromagnetic wavelength by the wavelet packet transform. Then, the Kirchhoff Model (KM) and Small Perturbation Method (SPM) were used to estimate the backscattering coefficient of the large-scale and small-scale roughness respectively. Moreover, the 'tilting effect' caused by the slope of large-scale roughness should be corrected when we calculated the backscattering contribution of the small-scale roughness. Backscattering coefficient of the MTSM was the sum of backscattering contribution of both scale roughness surface. The MTSM was tested and validated by the advanced integral equation model (AIEM) for dielectric randomly rough surface, the results indicated that, the MTSM accuracy were in good agreement with AIEM when incident angle was less than $30^{\circ}\left(\theta_{i}<30^{\circ}\right)$ and the surface roughness was small $(k s=0.354)$.
\end{abstract}

\section{INTRODUCTION}

The research on the scattering model for the randomly rough surface is an important part of microwave remote sensing theory. Several theoretical scattering models have been proposed for bare soil surface in past decades (Valenzuela, 1967; Ulaby et al., 1978; Jin, 2008). However, the scattering from a randomly rough surface is a very complex problem which does not admit exactly closed-formed solution from the Maxwell equations. Hence some of the approximate analytic solutions are possible only when the geometric scales of the surface are limited in the valid ranges. There are three representative approximate models: Kirchhoff Model (KM) (Beckmann, 1963), Small Perturbation Model (SPM) (Fung, 1967) and Integral Equations Model (IEM) (Fung, 1992). In the broad sense, KM is best suit for relatively rough surface (large-scale roughness surface), it is only valid when the electromagnetic wavelength is much smaller than the rough surface curvature radius. Meanwhile, the root mean square height $(s)$ and the associated correlation length $(l)$ which defines the surface roughness statistically are larger than the wavelength. SPM requires the relatively smooth surface (smallscale roughness surface), it is only effective when both of $\mathrm{s}$ and $l$ are smaller than the electromagnetic wavelength. However, natural surface contains various proportion of roughness, and it may not satisfy the ranges of validity conditions of KM or SPM. The integral equations model (IEM) proposed by Fung (1992) has implemented to improve ranges of applicability of the scattering models and gets a well accuracy. The basic theory of IEM is dividing the surface scattering field into two parts, the one is to keep the Kirchhoff field (the near field of tangent plane) as the initial solution, the other is to introduce the compensation field, which used to correct the Kirchhoff field.
IEM extends the valid rang of the theoretical scattering model and fills up parts of the gap between KM and SPM, therefore, it is the most popular method used in calculation of electromagnetic scattering of natural surface. Later, There are two important improvements on IEM accomplished by $\mathrm{Wu}(\mathrm{Wu}$ et al., 2001; 2004), which make the model more accurate in simulating the randomly rough surface. So IEM upgrades to Advanced Integral Equations Model (AIEM).

However, natural surface may be composed with complex mixture of large-scale roughness and small-scale roughness, such as ocean surface on which the small wave covers the large wave, or some block of clods with small granular soil on it. So in order to describe the electromagnetic scattering properties of this two-scale rough surface, we need to calculate the scattering effect of both sizes of the roughness. Ulaby (1981) firstly presented two-scale model (TSM) and attempted to solve the problem. In TSM, The randomly rough surface is assumed to be a combination of a slowly undulating surface that satisfies the $\mathrm{KM}$ condition and a small perturbation surface for which SPM is valid. When the incident angle is near normal $\left(0^{\circ}<\theta_{i}<25^{\circ}\right)$, the scattering is dominated by the large-scale surface roughness. While at large incident angles $\left(\theta_{i}>25^{\circ}\right)$, the scattering is dominated by small-scale surface roughness which is tilted by the slope of the large-scale surface roughness; So SPM is used to account for the tilting effect by introducing a local coordinate system and the scattering coefficient is calculated by considering both the large-scale and small-scale roughness. Brown proposed another two-scale model (Brown, 1978), a kind of Fourier function is used to transform the surface height to spectrum domain for a perfectly Gaussian surface, where the surface is filtered in its height spectral domain by a low pass

\footnotetext{
* Corresponding author
} 
filter. The small-scale roughness represented by the highfrequency spectral region and a first-order perturbation solution of Burrow's model (Burrows, 1967) is an adequate description of its scattering properties. The large-scale roughness represented by the low-frequency spectral region is assumed to be sufficiently smooth so as to form the unperturbed surface, and a physical optics approaches for determining the scattering field. The final result is the sum of the contribution of both scale surface roughness.

\section{SURFACE ROUGHNESS DECOMPOSITION BY WAVELET PACKET}

\subsection{Surface description}

The randomly rough surface is assumed to be a Gaussian stationary stochastic process. For any point $(x, y)$ on the surface, its surface height $(\mathrm{z}(x, y)$ is statistical variable which has the following statistical characteristics

$$
\begin{gathered}
E\langle z(x, y)\rangle=0 \\
E\left\langle z^{2}(x, y)\right\rangle=s^{2} \\
p(z(x, y))=\frac{1}{\sqrt{2 \pi} s} \exp \left\{-\frac{z(x, y)^{2}}{2 s^{2}}\right\}
\end{gathered}
$$

where $E<>$ is the ensemble average; $s$ is RMS of surface height; $p(z(x, y))$ is probability distribution function and $z(x, y)$ obeys the Gaussian distribution.

According to the so called composite surface scattering theory (Burrows, 1973) and the stochastic process theory (Mario, 1996; Fang, 2009), a Gaussian stationary stochastic process can be decomposed into two independent Gaussian stationary stochastic processes. So the surface height $z(x, y)$ may be rearranged into the following form (Fig. 1)

$$
z(x, y)=z_{k}(x, y)+z_{s}(x, y)
$$

where $z_{k}(x, y)$ is the height of large-scale roughness at $(x, y)$ and $z_{s}(x, y)$ is the height of small-scale roughness.

Since $z_{k}(x, y)$ and $z_{s}(x, y)$ are independent, the two-scale surface height spectrum $S\left(k_{x}, k_{y}\right)$ may be given as follows

$$
S\left(k_{x}, k_{y}\right)=S_{s}\left(k_{x}, k_{y}\right)+S_{k}\left(k_{x}, k_{y}\right)
$$

where $S_{k}\left(k_{x}, k_{y}\right)$ and $S_{s}\left(k_{x}, k_{y}\right)$ are the height spectrum for $z_{k}(x, y)$ and $z_{s}(x, y) ; k_{x}, k_{y}$ are wave number of the electromagnetic field incident upon the rough surface along the $\mathrm{x}$ and $\mathrm{y}$ directions respectively.

Eq. (4) and Eq. (5) along with the fact that $z_{k}(x, y)$ and $z_{s}(x, y)$ are zero-mean Gaussian are crucial to the development to follow. If $z(x, y)$ is non-Gaussian, it is not clear that the densities of $z_{k}(x, y)$ and $z_{s}(x, y)$ and their derivatives can be uniquely defined (Beckmann, 1975). More importantly, the spectral dichotomy as given by Eq.(5) may be invalid.

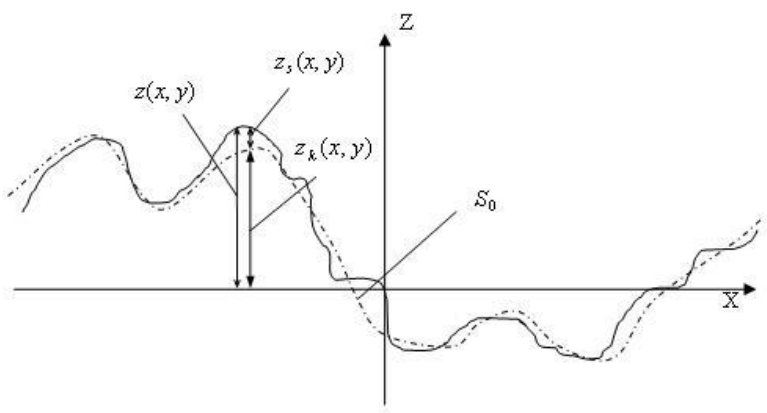

Fig.1 The decompose of two-scale roughness surface, the solid line represents the surface of $z(x, y)$, and the dotted line $\mathrm{S}_{0}$ is the large-scale surface $\left(z_{k}(x, y)\right)$ in X-Z plane

\subsection{Surface roughness decomposition by wavelet packets}

Wavelet is a time-frequency representation that has been used successfully in a broad range of applications, signal analysis in particular (Chui, 1992; Hilton et al., 1994). Wavelet packet is the development of wavelet multi-resolution analysis (MRA), it is a generalization of the wavelet transform that allows for arbitrary tree-shape bandpass filtering and can be adapted to the characteristics of the particular signal being analyzed.

The details of wavelet transform can be found in a number of literatures (Chui, 1992; Strang, 1996; Nielsen, 1996). In wavelet Multi-Resolution Analysis (MRA), the Hilbert space $\left(L^{2}(R)\right)$ is separated to the orthogonal sum of all the subspaces

$$
L^{2}(R)=\oplus W_{j}, j \in Z
$$

where $W_{j}$ is the subspaces of wavelet basis function $\varphi(t)$. In order to develop the frequency resolution, we can further divide wavelet subspace $W_{j}$ in accordance with the binary frequency segment. So the wavelet scaling subspace $V_{j}$ and $W_{j}$ can be jointed and represented by a new space $U_{j}^{n}(n \in N)$. If we suppose $U_{j}^{0}=V_{j}$ and $U_{j}^{1}=W_{j}$, as the formulation of orthogonal decomposition of $L^{2}(R), V_{j+1}=V_{j} \oplus W_{j}$ can be written as the decomposition of $U_{j}^{n}$

$$
U_{j+1}^{0}=U_{j}^{0} \oplus U_{j}^{1}
$$

Then, $U_{j}^{n}$ and $U_{j}^{2 n}$ are defined as the wavelet subspace of $u_{n}(t)$ and $u_{2 n}(t)$ respectively, $u_{n}(t)$ should satisfy the following equations

$$
\begin{gathered}
u_{2 n}(t)=\sqrt{2} \sum_{k \in Z} g(k) u_{n}(2 t-k) \\
u_{2 n+1}(t)=\sqrt{2} \sum_{k \in Z} h(k) u_{n}(2 t-k)
\end{gathered}
$$

where $g(k)$ and $h(k)$ are lowpass filter coefficient and highpass filter coefficient, and they are related by

$$
h(k)=(-1)^{k} g(1-k)
$$

When $n=0$, Eq. (8) can be changed to

$$
u_{0}(t)=\sqrt{2} \sum_{k \in Z} g(k) u_{0}(2 t-k)
$$




$$
u_{1}(t)=\sqrt{2} \sum_{k \in Z} h(k) u_{0}(2 t-k)
$$

The scaling function $\varphi(t)$ and basis function $\phi(t)$ in wavelet MRA are as follows

$$
\begin{aligned}
\varphi(t) & =\sqrt{2} \sum_{k \in Z} g(k) \varphi(2 t-k) \\
\phi(t) & =\sqrt{2} \sum_{k \in Z} h(k) \varphi(2 t-k)
\end{aligned}
$$

Comparing Eq. (10) with Eq. (11), we can find that $u_{0}(t)$ and $u_{1}(t)$ degenerated to $\varphi(t)$ and $\phi(t)$ respectively. $\left\{u_{\mathrm{n}}(t)\right\}$ is called the orthogonal wavelet packet determined by $u_{0}(t)=\varphi(t)$. Therefore, the wavelet decomposition is the special column of wavelet packet. When $n=0$, we use $u_{0}(t)$ and $u_{1}(t)$ as the subspace of wavelet packet, the wavelet packet transform changes to wavelet transform. On the other hand, if we go on decomposing the high-frequency spectral by MRA, the wavelet transform becomes wavelet packet transform.

There are several steps to decompose the surface roughness by wavelet packet in this paper.

1) The random surface height $z(x, y)$ are transformed to spectral domain by wavelet packet transform and the height spectral function is given by $S\left(k_{x}, k_{y}\right)$.

2) A free wave number $k_{d}$ is chose to split the roughness, so the height spectrum for $z_{s}(x, y)$ is $S_{s}\left(k_{x}, k_{y}\right)$, for $\left(\left|k_{x} \geq k_{d}\right| \cup\left|k_{y} \geq k_{d}\right|\right)$, while the height spectrum for $z_{k}(x, y)$ is $S_{k}\left(k_{x}, k_{y}\right)$, for $\left|k_{x}<k_{d}\right| \cap\left|k_{y}<k_{d}\right|$. Then, the surface height spectrum can be expressed as Eq. (5).

3) An inverse transform of wavelet packet is used to change $S_{s}\left(k_{x}, k_{y}\right)$ to the height function $z_{s}(x, y)$ of small-scale roughness, then we judge whether the RMS height $s_{s}$ and correlation length $l_{s}$ of $z_{s}(x, y)$ are agreed with the ranges of validity of the SPM $\left(k s_{s}<0.3, s_{s} l_{s}<0.21\right)$ (Fung, 1974). If not, going to the step 2 and reselecting the $k_{d}$.

4) When $k_{d}$ is determined, $S_{s}\left(k_{x}, k_{y}\right)$ and $S_{k}\left(k_{x}, k_{y}\right)$ can be calculated. Both scale of the roughness will be obtained by inverse transform of wavelet packet. So the surface roughness is divided to two parts successfully.

\section{THE SCATTERING COEFFICIENT OF THE MTSM}

\subsection{The scattering field of two-scale model}

In this paper, the equation of far-zone of electromagnetic scattering is used to derive the formula of MTSM. In accordance with the Stratton-Chu Equation (Ulaby et al., 1981), the far-zone scattered electric field is given in terms of the tangential electric and magnetic fields by

$$
\sigma_{p q}^{o}\left(\theta_{i}\right)=\sigma_{p q}\left(\theta_{i}\right)^{K A}+\sigma_{p q}^{(1)}\left(\theta_{i}\right)^{S P M}
$$

where $\sigma_{p q}\left(\theta_{i}\right)^{K A}$ is the scattering contribution of large-scale roughness at $\theta_{i}$ and $\sigma_{p q}{ }^{(1)}\left(\theta_{i}\right)^{S P M}$ is the first-order solutions of SPM for small-scale roughness. Because the small-scale roughness lay on the top of the large-scale roughness, which is so called 'tilting effect', we need to calculate the ensemble average of SPM to correct the 'tilting effect', then

$$
\sigma_{p q}^{o}\left(\theta_{i}\right)=\sigma_{p q}\left(\theta_{i}\right)^{K A}+\left\langle\sigma_{p q}{ }^{(1)}\left(\theta_{i}\right)^{S P M}\right\rangle
$$

At next, we will calculate the solution of $\sigma_{p q}\left(\theta_{i}\right)^{K A}$ and $\left\langle\sigma_{p q}{ }^{(1)}\left(\theta_{i}\right)^{S P M}\right\rangle$ respectively.

\subsection{Kirchhoff Scattering Field}

The KM applies tangent plane approximation to calculate the surface fields. This is valid if the radius of curvature is larger than a wavelength. When the surface roughness is large relatively $\left(k l>6\right.$ and $l^{2}>2.76 s \lambda$ ) (Ulaby et al., 1981), the KM reduces to the Geometric Optics Model by phase approximation, the backscattering coefficient is given by (Burrows, 1973)

$$
\sigma_{p q}\left(\theta_{i}\right)^{K A}=\frac{\left|\Gamma_{p q}(0)\right|^{2}}{2 m^{2} \cos ^{4} \theta_{i}} \exp \left(-\frac{\tan ^{2} \theta_{i}}{2 m^{2}}\right)
$$

where $m$ is the average slope and $m=\sqrt{2} s / l$ for the Gaussian surface; $\Gamma_{p q}(0)$ is the Fresnel reflection coefficient in the normal direction $\left(\theta_{i}=0\right)$, if $p \neq q, \sigma_{p q}\left(\theta_{i}\right)^{K A}=0$, else $\Gamma_{p q}(0)$ is given by

$$
\Gamma_{h h}(0)=\Gamma_{v v}(0)=\left|\frac{1-\sqrt{\varepsilon}}{1+\sqrt{\varepsilon}}\right|
$$

where $\varepsilon$ is dielectric constant.

When $0.05 \lambda<s<0.15 \lambda, l>\lambda$ and $m<0.25$, the KM reduces to the Physic Optics Model by scalar approximation, the backscattering coefficient is given by (Burrows, 1973)

$$
\begin{aligned}
& \sigma_{p q}\left(\theta_{i}\right)^{K A}=k^{2} \cos ^{2} \theta_{i} \Gamma_{p q}\left(\theta_{i}\right) \exp \left(-\left(2 k s \cos \theta_{i}\right)^{2}\right) \\
& \sum_{n=1}^{\infty} \frac{\left(2 k s \cos \left(\theta_{i}\right)\right)^{2 n}}{n !} W^{n}\left(2 k \sin \left(\theta_{i}\right), 0\right)
\end{aligned}
$$

where $W^{n}\left(2 k \sin \theta_{i}, 0\right)$ is the roughness spectrum of the surface related to the $n$-th power of the surface correlation function by the Fourier transform. For the Gaussian surface, $W^{n}\left(2 k \sin \theta_{i}, 0\right)$ is

$$
W^{n}\left(2 k \sin \left(\theta_{i}\right), 0\right)=\frac{l^{2}}{n} \exp \left\{-\frac{\left(k l \sin \theta_{i}\right)^{2}}{n}\right\}
$$

$\Gamma_{p q}\left(\theta_{i}\right)$ is the Fresnel reflection coefficient and it is denoted

$$
\begin{aligned}
\Gamma_{h h}\left(\theta_{i}\right) & =\left|\frac{\cos \theta_{i}-\sqrt{\varepsilon-\sin ^{2} \theta_{i}}}{\cos \theta_{i}+\sqrt{\varepsilon-\sin ^{2} \theta_{i}}}\right|^{2} \\
\Gamma_{v v}\left(\theta_{i}\right) & =\left|\frac{\varepsilon \cdot \cos \theta_{i}-\sqrt{\varepsilon-\sin ^{2} \theta_{i}}}{\varepsilon \cdot \cos \theta_{i}+\sqrt{\varepsilon-\sin ^{2} \theta_{i}}}\right|^{2}
\end{aligned}
$$


In the two-scale surface, because the large-scale roughness is covered by small-scale roughness, the Fresnel reflection coefficient in KM should be modified (Valenzuela, 1978)

$$
\begin{aligned}
& \left\langle\Gamma_{p q}\right\rangle=\Gamma_{p q}\left(\theta_{i}\right)\left[1-2 k \sin \theta_{i}\right. \\
& \left.\int_{-\infty}^{\infty} \int_{-\infty}^{\infty} W\left(u-k \sin \theta_{i}, v\right) \times K_{p q}(u, v) d u d v\right]
\end{aligned}
$$

where $\Gamma_{p q}$ is the corrected Fresnel reflection coefficient, $K_{p q}(u, v)$ is the KM filed coefficient, and $W\left(u-k \sin \theta_{i}, v\right)$ is the roughness spectrum.

\subsection{Small Perturbation Scattering Field}

The SPM is valid for the rough surface with small RMS height and small slope, its effective applying range are $k s<0.3$, and $s / l<0.21$. According to the former analysis in Eq. (27), the firstorder solutions of SPM is given by (Ulaby, 1981)

$$
\begin{aligned}
& \sigma_{p q}{ }^{(1)}\left(\theta_{i}\right)^{S P M}=8 k^{4} s^{2} \cos ^{4} \theta_{i}\left|a_{p q}\left(\theta_{i}\right)\right|^{2} \\
& W\left(2 k \sin \theta_{i}, 0\right)
\end{aligned}
$$

where $a_{p q}\left(\theta_{i}\right)$ is the polarized amplitude coefficient in SPM, and

$$
\begin{gathered}
a_{h h}\left(\theta_{i}\right)=\frac{\varepsilon_{s}-1}{\left(\cos \theta_{i}+\sqrt{\varepsilon_{s}-\sin ^{2} \theta_{i}}\right)^{2}} \\
a_{v v}\left(\theta_{i}\right)=\left(\varepsilon_{s}-1\right) \frac{\sin ^{2} \theta_{i}-\varepsilon_{s}\left(1+\sin ^{2} \theta_{i}\right)}{\left(\varepsilon_{s} \cos \theta_{i}+\sqrt{\varepsilon_{s}-\sin ^{2} \theta_{i}}\right)^{2}}
\end{gathered}
$$

In the two-scale surface, small-scale roughness lays on the large-scale one, so we need to correct the 'tilting effect'. $z_{k x}$ and $z_{k y}$ are supposed as the slope of the large-scale roughness $\left(z_{k}\right)$ in the direction of $\mathrm{x}$-axis and $\mathrm{y}$-axis respectively, and $P_{\theta i}\left(z_{k x}, z_{k y}\right)$ is the probability distribution function of $z_{k x}$ and $z_{k y}$. A simply rough surface is used to explain the probability distribution function by Fig. 3 , in which a simple rough surface is composed by three lines $l_{1}, l_{2}, l_{3}$ in a one-dimensional plane $x-z, z_{x 2}$ is the slope of $l_{2}$ and $P\left(z_{x 2}\right)$ is the probability distribution function of $z_{x 2}$. In the vertical direction $\hat{z}, P\left(z_{x 2}\right)$ can be written as (Jin, 1993)

$$
\begin{aligned}
P\left(z_{x 2}\right) & =\frac{\hat{l}_{2} \hat{n}_{2} \cdot \hat{z}}{\left(\hat{l}_{1} \hat{n}_{1}+\hat{l}_{2} \hat{n}_{2}+\hat{l}_{3} \hat{n}_{3}\right) \cdot \hat{z}}=\frac{\hat{l}_{2} \hat{n}_{2} \cdot \hat{z}}{L} \\
L & =\left(\hat{l}_{1} \hat{n}_{1}+\hat{l}_{2} \hat{n}_{2}+\hat{l}_{3} \hat{n}_{3}\right) \cdot \hat{z}
\end{aligned}
$$

In the direction of incident $\theta_{i}, P\left(z_{x 2}\right)$ is given by

$$
\begin{aligned}
P_{\theta i}\left(z_{x 2}\right) & =\frac{\hat{l}_{2} \hat{n}_{2} \cdot \hat{k}_{i}}{\left(\hat{l}_{1} \hat{n}_{1}+\hat{l}_{2} \hat{n}_{2}+\hat{l}_{3} \hat{n}_{3}\right) \cdot \hat{k}_{i}}=\frac{\hat{l}_{2} \hat{n}_{2} \cdot \hat{k}_{i}}{L_{\theta i}} \\
L_{\theta i} & =\left(\hat{l}_{1} \hat{n}_{1}+\hat{l}_{2} \hat{n}_{2}+\hat{l}_{3} \hat{n}_{3}\right) \cdot \hat{k}_{i}
\end{aligned}
$$

where $\hat{k}_{i}$ is the unit vector in the incident direction. From Eq. (37) and Eq. (39), we have

$$
P_{\theta i}\left(z_{x 2}\right)=\frac{L}{L_{\theta i}} \cos \theta_{i}\left(1+z_{x 2} \tan \theta_{i}\right) P\left(z_{x 2}\right)
$$

There are only three roughnesses in Fig. 3, so the sum of the probability distribution function is 1 , that is

$$
\sum_{m=1}^{3} P_{\theta i}\left(z_{x m}\right)=1
$$

Then,

$$
\frac{L}{L_{\theta i}} \cos \theta_{i}=1
$$

As a result, when it comes to the two-dimensional random surface, $P_{\theta i}\left(z_{k x}, z_{k y}\right)$ can be written as

$$
P_{\theta i}\left(z_{k x}, z_{k y}\right)=\left(1+z_{k x} \tan \theta_{i}\right) P\left(z_{k x}, z_{k y}\right)
$$

where $P\left(z_{k x}, z_{k y}\right)$ is the probability distribution function of the large-scale roughness, and it obeys a $\left(0, \sigma_{z k}^{2}\right)$ Gaussian distribution

$$
P\left(z_{k x}, z_{k y}\right)=\frac{1}{2 \pi \sigma_{Z k}{ }^{2}} \exp \left\{-\frac{z_{k x}{ }^{2}+z_{k y}{ }^{2}}{2 \sigma_{Z k}{ }^{2}}\right\}
$$

So the second term in Eq. (28) is given by (Jin, 1993)

$$
\begin{aligned}
& <\sigma_{p q}{ }^{(1)}\left(\theta_{i}\right)>^{S P M}=\int_{-\infty}^{\infty} \int_{-\cot \theta_{i}}^{\infty}\left(\hat{p} \cdot \hat{p}^{\prime}\right)^{4} \sigma_{p q}{ }^{(1)}\left(\theta_{i}^{\prime}\right) \\
& \left(1+z_{k x} \tan \theta_{i}\right) P\left(z_{k x}, z_{k y}\right) d z_{k x} d z_{k x}
\end{aligned}
$$

where $\hat{p}$ and $\hat{p}^{\prime}$ are the unit polarized vector of basic coordinate system and local coordinate system respectively. $\theta_{i}^{\prime}$ is the local incident angle. Due to avoid self-shadowing effect, the integral limit of $z_{k x}$ in Eq. (46) is from $-\cot \theta_{i}$ to $\infty$.

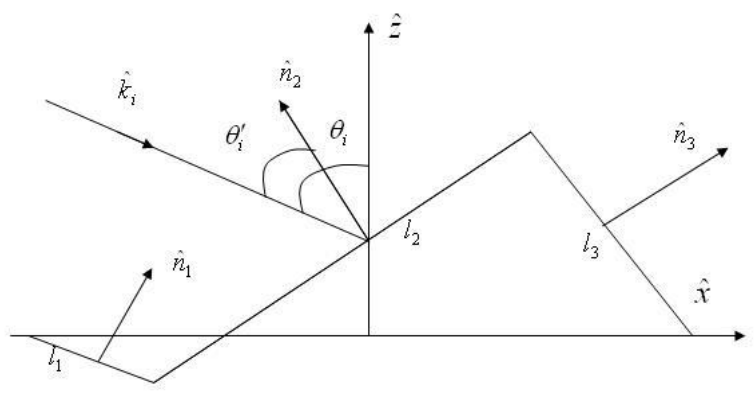

Fig. 3 The probability distribution of gradient in geometric explanation (Jin, 1993)

\section{VALIDATION}

The AIEM, which is the improvement of IEM, was adopted to verify the MTSM in this paper. With the widest range of applicability, the AIEM is the most popular method used in calculating the surface scattering in contrast to other models. In AIEM, the single scattering term is given by: 


$$
\begin{aligned}
& \sigma_{p q s}=\frac{k_{1}^{2}}{2} \exp \left(-s^{2}\left(k_{z}{ }^{2}+k_{s z}{ }^{2}\right)\right) \sum_{n=1}^{\infty} \frac{s^{2 n}}{n !}\left|I_{p q}{ }^{n}\right|^{2} \\
& W^{n}\left(k_{s x}-k_{x}, k_{s y}-k_{y}\right)
\end{aligned}
$$

where $W^{n}\left(k_{s x}-k_{x}, k_{s y}-k_{y}\right)$ is the roughness spectrum of the surface related to the $n$-th power of the surface correlation function by the Fourier transform. $f_{p q}$ and $F_{p q}$ are the Kirchhoff coefficient and the complementary field coefficient, respectively. $k_{z}=$ $k \cos \theta_{i} ; k_{s z}=k \cos \theta_{s} ; k_{x}=k \sin \theta_{i} \cos \varphi_{i} ; k_{s x}=k \sin \theta_{s} \cos \varphi_{s} ; k_{y}=$ $k \sin \theta_{i} \sin \varphi_{i} ; k_{s y}=k \sin \theta_{s} \sin \varphi_{s}$

The inputs of simulations were set to: frequency $f=5.63 \mathrm{GH}$; complex dielectric constant $\varepsilon_{s}=19.54+3.08 i$; Incident angle $\theta_{i} \epsilon$ $\left[10^{\circ}, 70^{\circ}\right]$; three different Gaussian surfaces $(k s=0.354,1.061$, $2.352 ; k l=8.21$ ) were supposed to test the MTSM respectively. And the choice of wave number $k_{d}$ for splitting the roughness in MTSM should satisfy the following two requirements:

$$
\begin{aligned}
s_{s} & =\frac{s}{5}, \quad k s<1.5 \\
k s_{s} & =0.3, k s \geq 1.5
\end{aligned}
$$
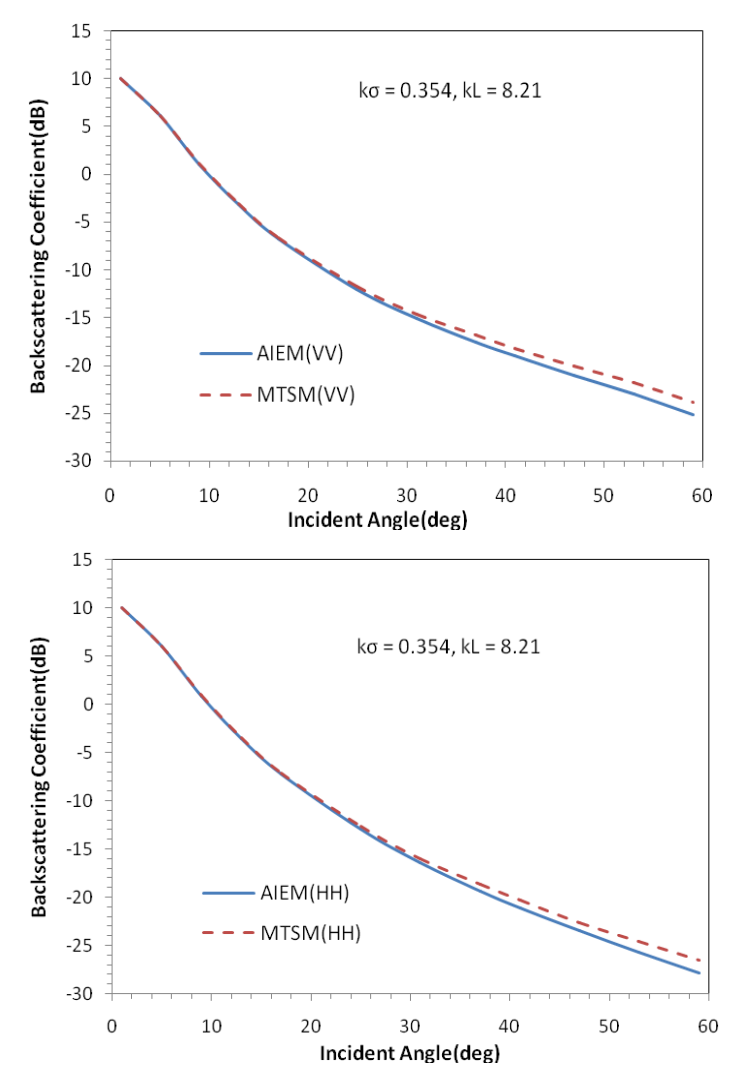

(a)
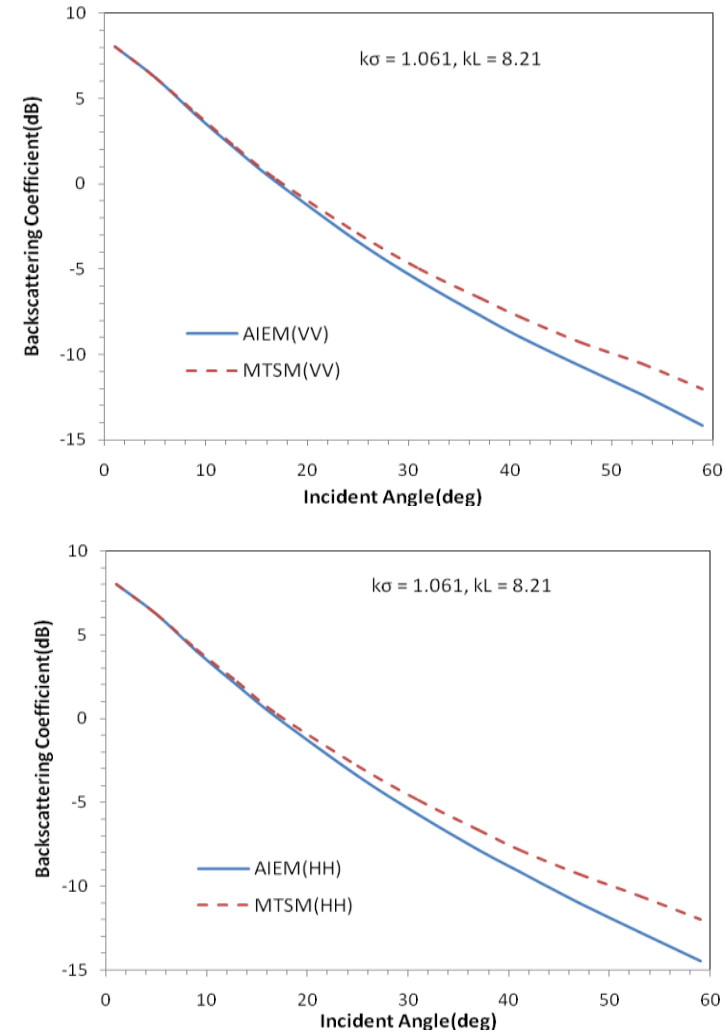

(b)
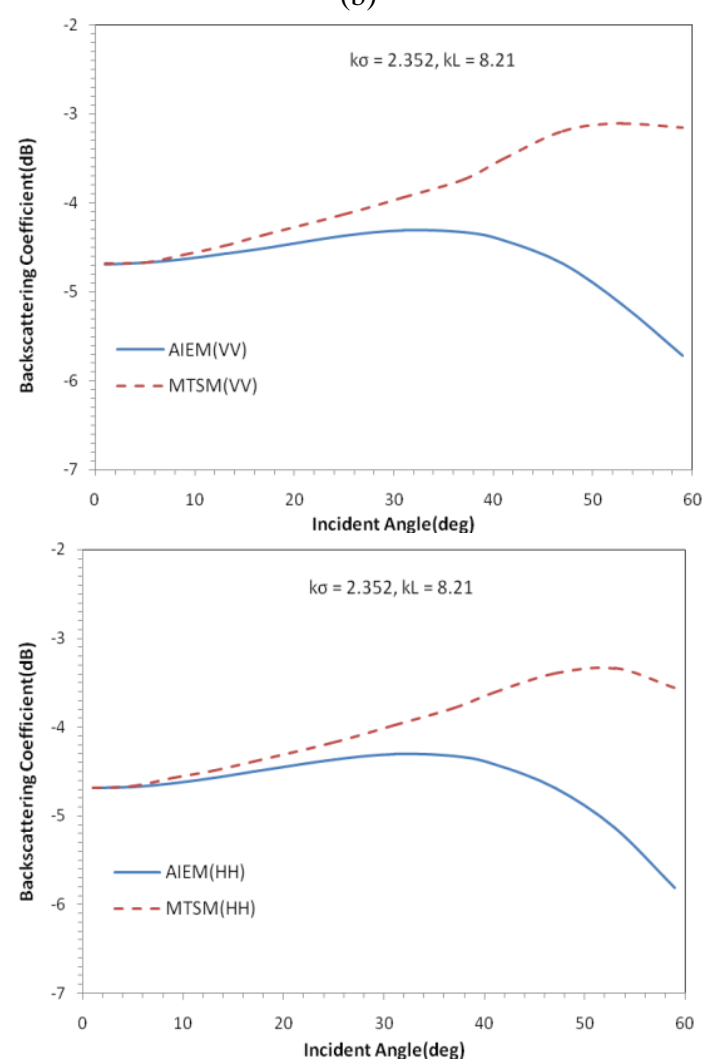

(c)

Fig. 4 Comparison of the backscattering coefficient predicated by the AIEM and MTSM with $k l=8.21, \theta_{i} \in\left[10^{\circ}, 70^{\circ}\right]$, and $\varepsilon_{s}$ $=19.54+3.08 i$ for different roughness: (a) $k s=0.354$, (b) $k s=$ 1.061 , and (c) $k s=2.352$ 
Fig. 4 shows scatter plots of backscattering coefficient estimated using the MTSM Versus AIEM on the three different roughness surfaces. The MTSM is in good agreement with the AIEM at small incident angles. When $\theta_{i}$ increases, the difference between them become larger and reach the largest value of $2.561 \mathrm{~dB}$ at $\theta_{i}$ $=60^{\circ}$ in $\mathrm{VV}$ polarization. In additional, when the surface roughness is small $(k s=0.354)$, it is clear that the MTSM give very accurate predictions, presenting less than $0.68 \mathrm{~dB}$ difference with the AIEM both for VV and $\mathrm{HH}$ polarization. As the surface roughness increases, we see that the results of the MTSM move far from the AIEM, especially in Fig. 4(c), their difference is more than $2 \mathrm{~dB}$ for both polarizations when $\theta_{i}>$ $45^{\circ}$. The estimation of MTSM in VV polarization is slightly closer to AIEM than in $\mathrm{HH}$ polarization, it indicates that the polarization manner has slightly influence on the MTSM. Therefore, MTSM can achieve well accuracy when both of the incident angle and surface roughness are small.

\section{CONLCUSION}

In this paper, a new model (MTSM) was proposed to describe the backscattering for a Gaussian-distributed rough surface. The surface height function $z(x, y)$ is transformed to frequency region by wavelet packet, and the surface height spectrum was split into two parts. The large-scale roughness corresponding to the low frequency portion of the spectrum $\left(k<k_{d}\right)$, is assumed that the Kirchhoff Model adequately describes the scattering from these height excursions. The small-scale roughness is represented by the high frequency portion of the total height spectrum $\left(k>k_{d}\right)$, it is described by a first order SPM. The key parameter $k_{d}$ for spectral dichotomy is determined by Eq. (47). Moreover, the 'tilting effect' caused by the large-scale roughness was corrected, and backscattering of the MTSM is the sum of both scale roughness surfaces. From the results of the numerical experiments, the following conclusions can be drawn:

1) It is a difficult task to measure the large-scale and small-scale roughness in traditional two-scale model. Wavelet packet transform is used to split the surface height spectrum into two parts, these makes the two-scale model useful in practice.

2) The comparison between the simulations of AIEM shows that a good agreement exists between the AIEM and MTSM when both of the incident angle and surface roughness are small The different polarization has slightly influence on the MTSM.

3) It should be noted that the MTSM was established to describe the electromagnetic scattering properties of typically two-scale land surface, and it was tested using AIEM model by numerical simulation only, its application to natural surface and its ranges of validity of surface roughness remains the subject of further study and is ongoing.

\section{REFERENCES}

Beckmann, P.A., Spozzochino, T., 1963. The Scattering of Electromagnetic Waves from Rough Surface. Macmillan, New York, pp. 121-133

Beckmann, P.A., 1975. Scattering by non-Gaussian surfaces. IEEE Transaction on Antennas and Propagation, 21(2), pp. 169-175.

Brown, G.S., 1978. Backscattering from a Gaussian distributed perfectly conducting rough surface. IEEE Transaction on Antennas and Propagation, 26(3), pp. 472-482.
Burrows, M.L., 1967. A reformulated boundary perturbation theory in electromagnetism and its application to a sphere. Canadian Journal of Physics, 45(5), pp. 1729-1743. Burrows, M.L., 1973. On the composite model for rough surface scattering. IEEE Transaction on Antennas and Propagation, 21(2), pp. 241-243.

Chui, C.K., 1992. An Introduction to Wavelets. Academic, New York, pp. 245-265

Fang, Z., 2011. Stochastic Processes. Science, Beijing, pp. 2335

Fung, A.K., 1967. Theory of cross-polarized power returned from a random surface. Applied Science Research, 18(1), pp. 50-60.

Fung, A.K., 1992. Backscattering from a randomly rough dielectric surface. IEEE Transaction on Geoscience and Remote Sensing, 30(2), pp. 356-369.

Hilton, M.L., Jawerth, B.D., 1994. Compressing still and moving images with wavelets, Multimedia Systems, 2(5): 218 227. DOI: $10.1007 / \mathrm{BF} 01215399$

Jin, Y.Q., 2008. Theory and Method of Numerical Simulation of Composite Scattering from the Object and Randomly Rough Surface. Science, Beijing, pp. 242-245

Mario, L., 1996. Applied Stochastic Processes. John Wiley and Sons, New York, pp. 112-132

Nielsen, N.H., Wickerhauser, M.V., 1996. Wavelets and timefrequency analysis. Proceeding of IEEE, 84(4), pp. 523-540.

Strang, G., Nguyen, T.Q., 1996. Wavelets and Filter Banks. Wellesley: Wellesley-Cambridge, London, pp. 5-8

Ulaby, F.T., Batlivala, P., Dobson, M., 1978. Microwave backscatter dependence on surface roughness, soil moisture and soil texture: Part 1-bare soil. IEEE Transaction on Geoscience Electronics, 16(4), pp. 286-295.

Ulaby, F.T., Moore, P.K., Fung, A.K., 1981. Microwave Remote Sensing, Vol II: Microwave Remote Sensing Fundamentals and Radiometry. Addison Wesley Publishing Company, London, pp. 287-289.

Valenzuela, G.R., 1967. Depolarization of EM waves by slightly rough surface. IEEE Transaction on Antennas and Propagation, 15(4), pp. 552-557.

Wright, J.W., 1968. A new model for sea clutter. IEEE Transaction on Antennas and Propagation, 16(2), pp. 217-223. Wu, T.D., Chen, K.S., Shi, J.C., 2001. A transition model for the reflection coefficient in surface scattering. IEEE Transaction on Geoscience and Remote Sensing, 39(9), pp. 2040-2050.

Wu, T.D., Chen, K.S., 2004. A reappraisal of the validity of the IEM model for backscattering from rough surface. IEEE Transaction on Geoscience and Remote Sensing, 42(4), pp. 743-753. 